\title{
Geomorphologie der alpinen Stufe des Chirripó in Costa Rica
}

\author{
Jorge Barquero \& LudWig Ellenberg *)
}

\begin{abstract}
Mountains, pre-glacial morphology, drainage patterns, glacial morphology, cirques, moraine ridges, periglacial features, Pleistocene, Holocene
\end{abstract}

Costa Rica

Kurzfassung: Im Relief der alpinen Stufe des Chirripó in Costa Rica sind über der heutigen Waldgrenze bei $3400 \mathrm{~m}$ Höhe drei Reliefgenerationen nachzuweisen.

Die erste ist vorkalt $\mathrm{z}$ e it li ch. In ihr wurden die Talzüge und der Verlauf der Wasserscheiden festgelegt. Die zweite ist $\mathrm{k}$ a l t z e i $\mathrm{t} \mathrm{ich}$. In ihr wurde das Relief am stärksten geprägt. Kargletscher mit Zungen in den Tälern vertieften und weiteten in allen Richtungen die Talschlüsse und Oberläufe der Täler. Einige während dieser Zeit entstandene Karlinge, Kare, Moränen und Rundhöcker wurden nach der Vereisung durch Periglazialprozesse in den Teilen überprägt, wo eine Schuttauflage vorhanden war.

Die dritte Reliefgeneration wird durch das he u t ig e $\mathrm{ProzeBgefüge}$ bestimmt. Es ist nur schwach entwickelt; die Formen verändern sich langsam. Lediglich leichte Nachtfröste, ausschließlich episodisch oberflächlich abfließendes Regenwasser und kleine Verwitterungsgeschwindigkeiten wirken nur langsam auf das Relief ein. Tiefer gelegene Bergstufen Costa Ricas sind geomorphologisch wesentlich aktiver. Die glazial entstandenen Formen blieben auch wesentlich besser erhalten als in höher aufragenden tropischen Gebirgen oder in den Hochgebirgsketten der gemäßigten Breiten. So hat sich hier eine vor allem kaltzeitlich gestaltete Bühne erhalten können - die einzige in Zentralamerika.

\section{[Geomorphology of the Alpine Stage of the Chirripó in the Cordillera de Talamanca in Costa Rica]}

Abstract: The relief features of the alpine stage in the Chirripó mountainous area in Costa Rica which are to be found above the altitude of $11,000 \mathrm{ft}$. have been formed by three different generations of geomorphological processes. The first one of importance was $\mathrm{p} \mathrm{r} \mathrm{e} \mathrm{g} \mathrm{l} \mathrm{a} \mathrm{c} \mathrm{i} \mathrm{a} \mathrm{l.} \mathrm{During} \mathrm{that}$ period the valleys and watersheds were initiated. The second one was of $\mathrm{gl} \mathrm{a} \mathrm{c} \mathrm{i} \mathrm{a} \mathrm{l} \mathrm{character.} \mathrm{During} \mathrm{the} \mathrm{Pleistocene}$ the relief features were subject of an intensive alteration.

*) Anschrift der Autoren: Lic. J. Barquero, Prof. Dr. L. ElLenBerg, Escuela de Ciencias Geográficas, Universidad Nacional, Apartado 86, Heredia, Costa Rica.
Coming from the cirques small glaciers with tongues down to perhaps $10,000 \mathrm{ft}$. had their part in forming the upper parts of the valleys into glacial throughs. Some of the cirques, moraine ridges, and roches moutonées have partially been changed after the glacial phase by the processes of solifluction. The third generation can be characterized by presently acting processes, but in fact they are not as active as in other Costa Rican mountain ridges or in lower stages of the Chirripó. Freezing actions as such are not hard, the superficial running off of the rain-water is not excessively heavy, and erosion inside the wide valleys is easy to observe but not very intense. The forms which are to be dated from the last glaciation are much better preserved here than in other tropical mountains, which reach into higher altitudes and are also much better than in mountain ridges of ex-tropical countries. In the Chirripó mountainous area of Costa Rica a nearly unchanged glacial stage is to be found - the unique of this kind between Colombia and Mexico.

\section{[Geomorfología del piso alpino del Chirripó en la Cordillera de Talamanca, Costa Rica]}

Resumen: En el piso alpino del Chirripó se observan tres generaciones de formación del relieve en las regiones más altas de $3400 \mathrm{~m}$. La primera es antes de la época glaciar, se formaron las direcciones de los valles y las divisorias. La segunda en la época glaciar es la más importante. Los glaciares han formado circos, valles en $U$, morrenas y rocas aborregadas. Inmediatamente después de la época glaciar esta región tenía influencia por procesos solifluidales; prueba son las terrazas pequeñas en unas pendientes. La tercera generación es la de hoy, con procesos en relación al clima reciente. Las formaciones por procesos de hielo son muy suaves, el agua en las pendientes existe solamente en periodos o episodios y el cambio por procesos fluviales recientes es mucho menor que en los pisos más bajos de la Cordillera de Talamanca.

El resultado de las observaciones en el piso alpino del Chirripó es que existe un relieve de origen glaciar, sin grandes cambios como consecuencia de los procesos recientes. Se puede observar la única región formada por glaciares entre Colombia y México con poca influencia por los procesos de los últimos 10000 años. 


\section{Einleitung, Stand der Bearbeitung und Fragestellung}

Bis vor 30 Jahren waren Vergletscherungsspuren in Zentralamerika unbekannt. Hinweise auf glaziale Gestaltung hatte man zwar in Kolumbien und Venezuela sowie in Mexiko gewonnen, doch klaffte hinsichtlich solcher Befunde eine $2000 \mathrm{~km}$ messende Lücke, obwohl die Berge in Guatemala (Volcán Tajumalco $4220 \mathrm{~m}$ ), Costa Rica (Volcán Irazú $3422 \mathrm{~m}$, Cerro Chirripó 3819 m) und Panamá (Volcán Chirriquí $3478 \mathrm{~m}$ ) hoch genug erschienen, daß sie während der pleistozänen Kaltzeiten nicht nur intensiver durch frost- und frostwechselinduzierte Prozesse umgestaltet wurden, sondern sich auch Gletscher aufbauen konnten. Die Entstehung der hohen Aufragungen, zumeist quartäre Vulkane, erschien zu jung und bei den nichtvulkanischen Bergen konnte angenommen werden, daß erst eine subrezente Hebung sie in die potentiell gletschergeprägten Höhenstufen angehoben hätten.

Erst anläßlich einer Überfliegung der Cordillera de Talamanca im S von Costa Rica fielen WEYL Mitte der 50-er Jahre die Glazialformen am Chirripó auf. Daß dies so spät geschah, läßt sich leicht erklären. Die Cordillera de Talamanca wurde bald nach der um 1560 erfolgenden Einwanderung galicischer und asturischer Siedler zu einem Rückzugsgebiet der indianischen Bevölkerung. Die Hänge sind unzugänglich steil (intensive tektonische Heraushebung, nah gelegene Erosionsbasis des Pazifiks und Atlantiks, hohe Niederschläge zwischen Mai und November, kurze Bäche und Flüsse mit steilem Längsprofil und Wasserfällen), Rutschungen an ihnen häufig (tief verwitterte Substrate bis etwa $2500 \mathrm{~m}$ Höhe, wasserbindende Böden, intensive Erdbebentätigkeit), Flußterrassen nur als schmale Leisten entwickelt (rasches Zurückschneiden der Terrassenhänge) und der Bau gesicherter Straßen deshalb fast unmöglich. Den europäischen Siedlern der Kolonialzeit standen geeignetere Naturräume zur Verfügung: das klimatisch angenehme Hochland des Valle Centrals, gemäßigt in den Temperaturen und regenreich bei kurzer Trockenperiode, also NWSpaniern durch die feuchte Variante eines Cs-Klimas wohl vertraut. Auch das relativ trockene und für Viehwirtschaft geeignete Tiefland von Guanacaste und der schmale Korridor vom Hochland zum Pazifik als Verbindung zwischen diesen Erschließungszentren waren wesentlich attraktiver. Auch heute zielt die costarricensische Binnenkolonisation nicht auf die Cordillera de Talamanca, sondern auf die peripheren Tiefländer. Die Cordillera im Landesinneren wurde erst dann wissenschaftlich bekannt, als die größten Teile Costa Ricas bereits bearbeitet waren.
Ein erstes Inventar und eine Interpretation der Ergebnisse erfolgte durch die Arbeit von WEYL. Diese Studien (WEYL 1955, 1956, 1957) wurden von HASTENRATH fortgeführt. Er vervollständigte die Übersichtskartierung, korrelierte die einzelnen Moränenstände, erklärte die geologisch bedingte Asymmetrie der Hänge und der Vergletscherungsspuren und wies auf das fast völlige Fehlen rezenter Periglazialformen hin (HASTENRATH 1973).

Eine junge Hebung des auf $9^{\circ} 30^{\prime} \mathrm{N}$ gelegenen Berggebietes ist zwar nachzuweisen, war aber für die Erklärung der pleistozän auf 3500 m Höhe befindlichen Schneegrenze unnötig. In den niederschlagsreichen Gebieten des tropischen Lateinamerikas sind tief reichende Gletscherstirnen die Regel und nicht die Ausnahme, wie die Arbeiten von SCHUBERT (1975) für die venezolanischen Anden, SCHUBERT (1976) für die Sierra de Perija und HeINE (1977) für Mexiko belegt haben.

Geomorphologisch gab es in der Cordillera de Talamanca seither keinen weiterführenden Beitrag. Die Publikation von BERGOEING (1977) stützt sich lediglich auf eine Interpretation von Luftbildern. In den letzten 25 Jahren und besonders, seit die obere Stufe des Nebelwaldes im August $1975 \mathrm{zu}$ einem $473 \mathrm{~km}^{2}$ großen Nationalpark erklärt werden konnte (BOZA \& MENDOZA 1981), wurden allerdings mehrere biologische Arbeiten durchgeführt (WEBER 1959; CHAVERRI et al. 1976; VAUGHAN et al. 1976).

In unserer Arbeit verfolgen wir zwei Ziele.

Erstens soll ein geomorphologisches Inventar der alpinen Stufe des Chirripó detailliert in einer Karte dargestellt werden: die präglazial angelegten Formen, das glazial gestaltete Relief und die danach eingetretenen Veränderungen.

Die Beantwortung der Frage, ob das Relief des Chirripó eine "glaziale Bühne in Formungsruhe" oder ein rezent gestaltetes Hochgebirge ist, beinhaltet das zweite Ziel. Die gegenwärtig gestaltenden Prozesse und ein Abschätzen ihrer Geschwindigkeit stehen dabei im Vordergrund der Beobachtung.

Dabei wird auf 15 Besteigungen des Massivs durch JORGE BARQUERO und eine gemeinsame Feldarbeit im Februar 1984 zurückgegriffen, wobei die Autoren von José VindaZ, BERND RAEDEL und GÜNTER LANGNER begleitet wurden.

\section{Geomorphologische Kartierung}

Abb. 1 stellt das Arbeitsgebiet in einer Übersicht dar. In ihr ist die glazial gestaltete Region über der 


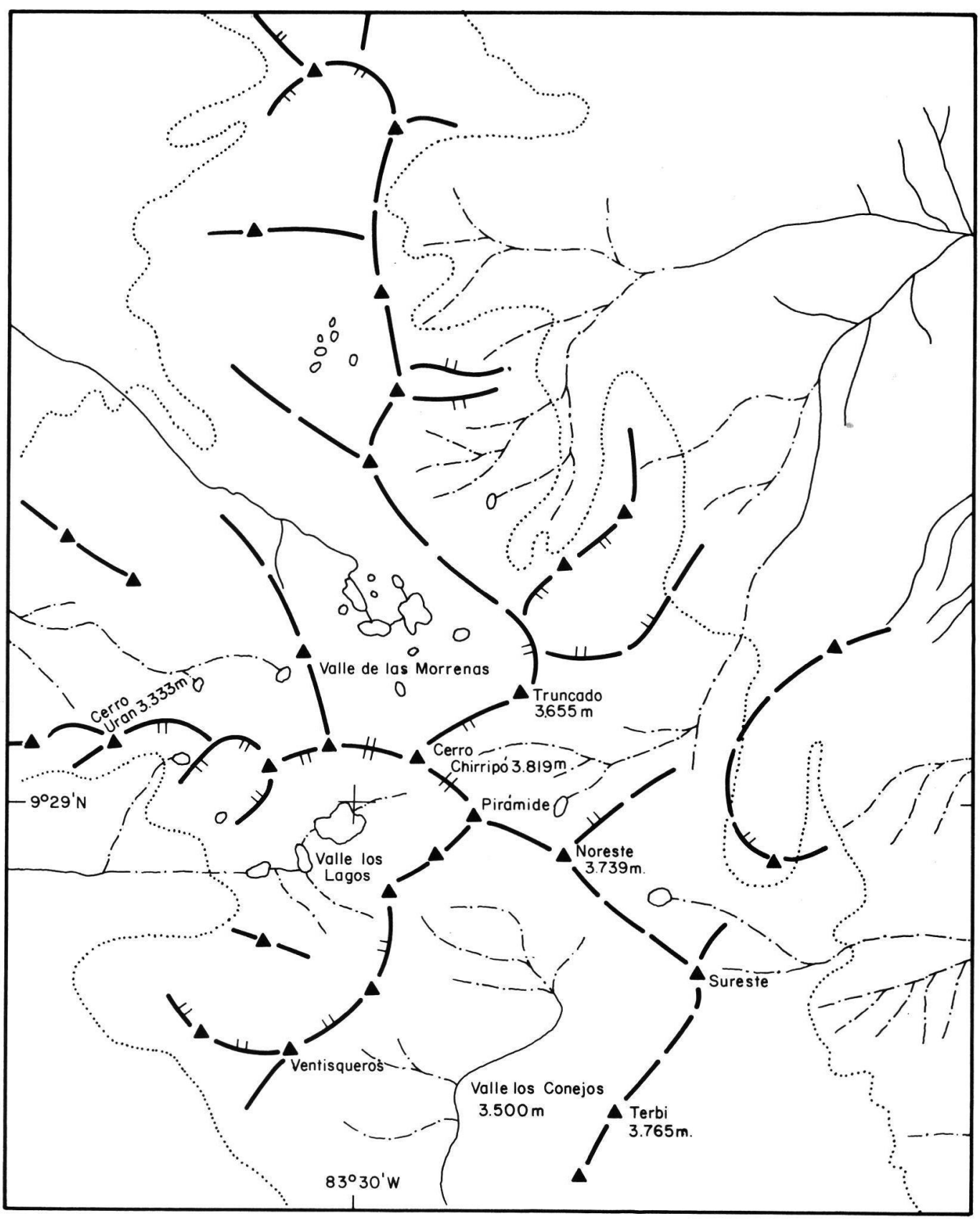

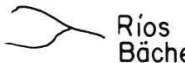

Bäche

.2. Quebradas intermitentes Saisonale Bäche

Po Lagunas

F Circos

Limitación arboreo Waldgrenze
Escala gráfica

Masstab der Zeichnung

0 I $2 \mathrm{Km}$

Abb. 1: Das Arbeitsgebiet in einer Übersicht. 

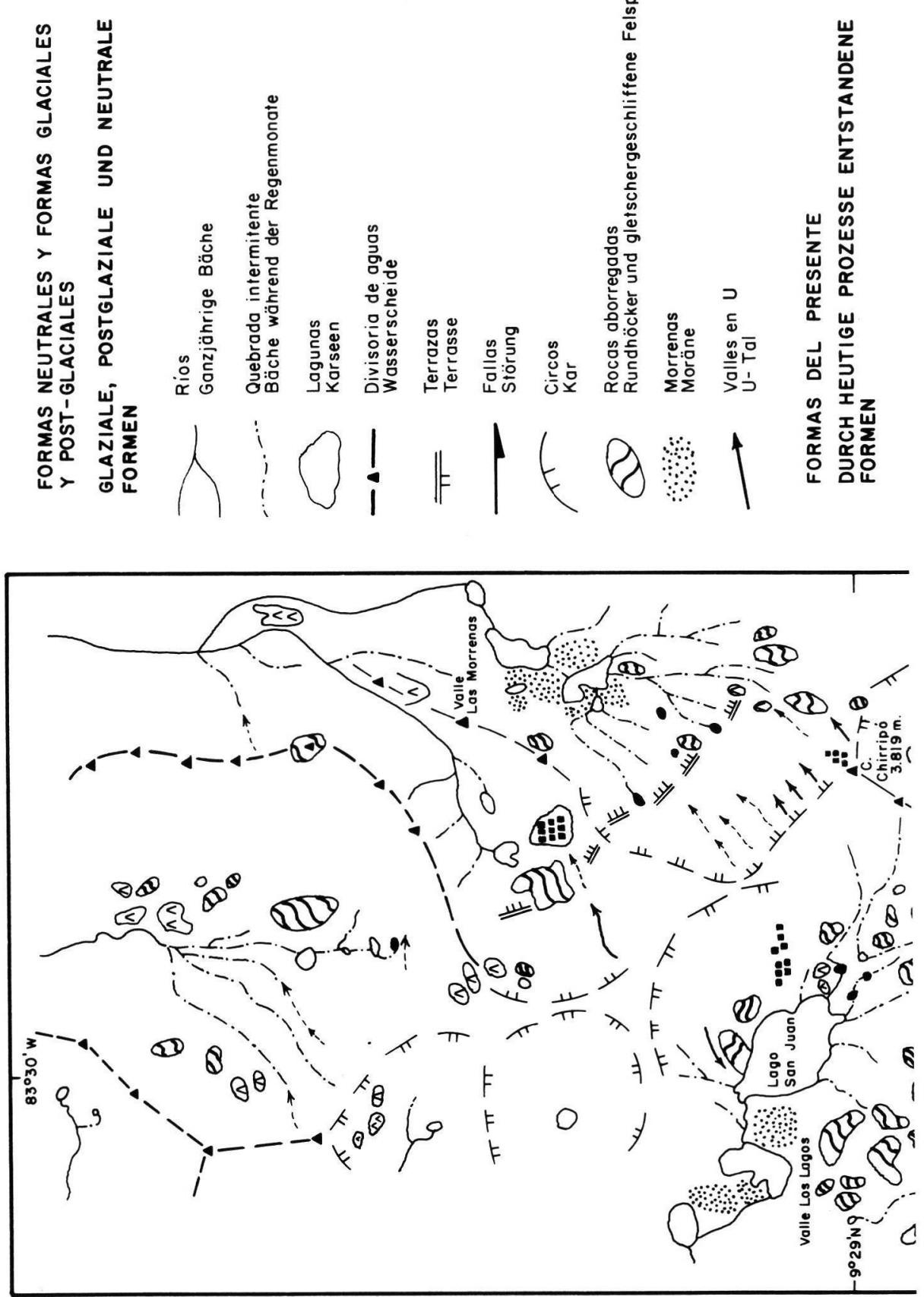

Abb. 2: Glaziale, postglaziale und neutrale Formen.

Waldgrenze umrissen. $3400 \mathrm{~m}$ Höhe kann als mittlerer Wert gelten für die Untergrenze der ehemaligen Vergletscherung; die tiefsten Gletscherzungen erreichten $3300 \mathrm{~m}$. Das glazial gestaltete Gebiet umfaßt den größten Teil der alpinen Stufe über der oberen
Waldgrenze. Folgende Fragen, die sich an die Arbeit von HASTENRATH (1973) anschließen ließen, blieben unbeantwortet: Wie alt sind die Moränen? Welchen Einfluß hatte die jüngste Hebung der Cordillera de Talamanca? 

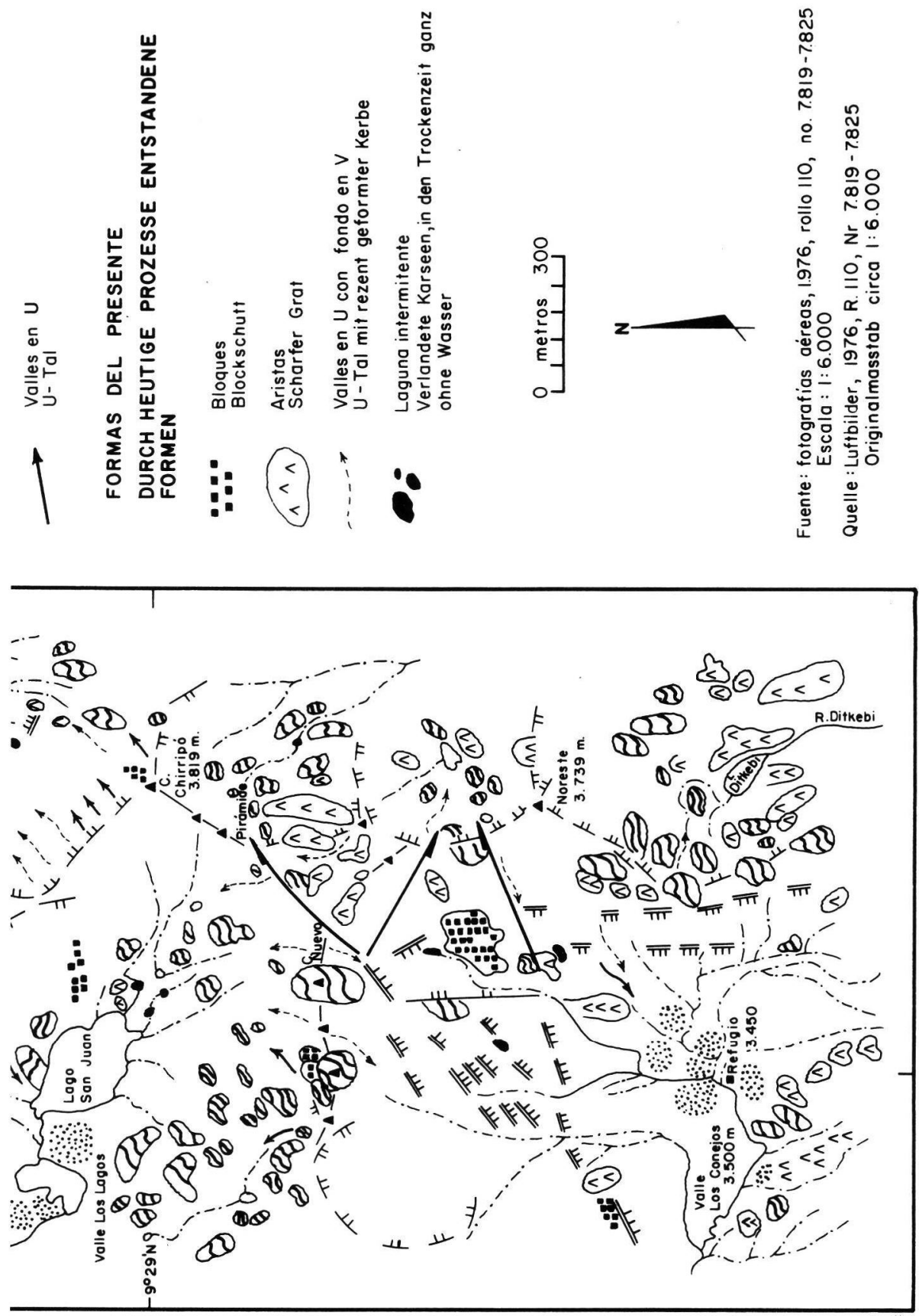

Abb. 2 greift einen kleinen Teil der alpinen Stufe heraus. Im Maßstab 1:12000 wurden charakteristische Kleinformen festgehalten. Im Valle de los Conejos und im Valle de los Lagos stützt sich die Darstellung auf mehrere Routenkartierungen. Sie wurden durch
Übersichten vom Chirripó Grande $(3819 \mathrm{~m})$ und mehreren Nebengipfeln ausgeweitet. Eine Interpretation der Luftbilder aus dem Jahre 1976 konnte zur Ergänzung verwendet werden. 
Gänzlich vegetationsfrei sind lediglich einige Steilwände aus tertiären Intrusivgesteinen (besonders Quarzdioriten), Blockhalden und Kuppen von Rundhöckern. Glazial gestaltete F e l s b u c k e l wurden auch dort kartographisch festgehalten, wo sie teilweise unter einer dünnen Schutt- und Bodendecke liegen, aber als Form zu erkennen sind. Sie machen $4 \%$ der kartierten Fläche aus, 0.14 von $3,6 \mathrm{~km}^{2}$. Die $\mathrm{M}$ or ä n e n sind weiter verbreitet als in der Kartendarstellung ersichtlich ist, da nur die als Vollform oder mächtige Schuttakkumulationen erkennbaren Teile eingezeichnet wurden. K a r e (Taf. 1, Fig. 1) sind über $3500 \mathrm{~m}$ Höhe reichlich vorhanden. Nur die größten und heute wassergefüllten weisen eine deutlich ausgeprägte Schwelle und einen versteilten, schuttarmen Talschluß auf. Einige der kleineren sind zu Auffangbecken eingeschwemmten Feinmaterials geworden und dadurch weitgehend verlandet. Sie treten während der Monate Dezember, Januar und Februar als trockene Schlammflächen, während der Regenmonate aber als flache $\mathrm{Te}$ i c h e in Erscheinung. Alle von den hochgelegenen glazial überprägten Talschlüssen radial ausgehenden Talzüge weisen $\mathrm{U}-\mathrm{Q}$ u e r s chnit te auf und sind bis etwa $3400 \mathrm{~m}$ Höhe nicht sehr steil. Innerhalb der Waldstufe allerdings steigt das Gefälle, ist ein V-Querschnitt die Regel und kann keine glaziäre Prägung nachgewiesen werden.

Die glazial entstandenen Formen können zwar der letzten Kaltzeit zugeordnet werden, doch ist eine genauere Einstufung noch nicht gelungen. Die Frische der Formen suggeriert einen Analogieschluß zu nördlich und südlich benachbarten Gebirgsvergletscherungen, die als spätglazial angesehen werden, wobei höhere Niederschläge für die Gletschervorstöße wichtiger waren als erniedrigte Temperaturen (SCHUBERT 1976; HEINE 1983).

Die in Abb. 2 dargestellten glazial entstandenen Formen werden ergänzt durch die ,neutralen” Reliefeinheiten, wie die W a s serscheiden, die auch schon vor der Vereisung und ebenfalls danach morphodynamisch aktiv waren. Außerdem sind die Formen hervorgehoben, die durch gegenwärtige Prozesse modelliert werden.

Am häufigsten kommen dabei schmale $\mathrm{T}$ a $1 \mathrm{k}$ e $\mathrm{r}$ b e n (Taf. 1, Fig. 2) vor. Sie sind Übertiefungen innerhalb der muldenförmigen Täler und Hangnischen. Sie können bis zu mehreren Metern Tiefe erreichen, bleiben aber immer schmal und setzen sich mit scharfen Kanten vom glazial gestalteten und solifuidal überprägten Talboden ab. Die meisten sind nur im Gelände selbst und nicht in Luftbildern zu erkennen, da die Schicht der Büsche sie von den Seiten her fast ganz überdeckt. Die Talkerben sind nach den einzel- nen Regengüssen kurzzeitig aktiv, werden aber von so kleinen Einzugsgebieten gespeist, daß ihre Einschneidungsgeschwindigkeit seit Beginn des heutigen Prozeßgefüges nur kleine Formen geschaffen hat. An schwach geneigten Hangpartien setzen die Kerben ganz aus und das abfließende Wasser benutzt den Boden der Hangmulden.

An mehreren Stellen wurden Blöcke durch Frostprozesse aus den Felswänden gelöst und bilden $\mathrm{S} t \mathrm{u}$ r z $\mathrm{h}$ a $\mathrm{ld} \mathrm{e} \mathrm{n}$. Eine flächige Frostschuttproduktion aber fehlt. Die Schuttzungen sind in der Regel schmal und nur wenige Meter lang. Sie werden von der Buschvegetation in ihrer hangabwärtigen Bewegung gestoppt. Die einzige große Blockhalde ist an eine Störung gebunden, wo die mechanische Verwitterung leichter ansetzen konnte als an den meisten anderen Stellen. Unbewachsener Hangschutt macht nur 0,6\% der kartierten Fläche aus.

Rezente Abspülung, Abbrechen von gelockerten Blöcken und Frostsprengung sind an den meisten $\mathrm{G} r$ a t e n erkennbar, ohne daß sie von kaltzeitlicher Formung präzise abzugrenzen wäre, da die Wasserscheiden dauernd schnee- und eisfrei blieben. An ihnen änderte sich nur die Intensität, nicht aber die Qualität der geomorphologischen Prozesse.

Nicht darzustellen ist in der Karte die seit der Klimaverbesserung eingesetzte $\mathrm{Boden} \mathrm{bild} \mathrm{ung}$. Sie ist deutlich langsamer als in der Waldstufe, wo unterhalb von $3000 \mathrm{~m}$ Höhe Felsuntergrund nur an frischen und tiefgreifenden Rutschungen hervortritt. In der alpinen Stufe hingegen sind Skelettböden mit einem sehr kleinen Anteil an Feinmaterial bestimmend. Freigelegte Felsbuckel verwittern nur langsam, wie viele gut erhaltene $\mathrm{Gle} \mathrm{t} \mathrm{s} \mathrm{ch}$ e $\mathrm{r} \mathrm{s} \mathrm{ch} \mathrm{l}$ iffe (Taf. 1, Fig. 3) belegen.

Ebensowenig sind Formen rezenter periglazialer Prägung in der Karte zu vermerken. Es kommen lediglich $\mathrm{k}$ a m meisind u zi e r e B od e n bewe $\mathrm{g} \mathrm{u} \mathrm{n} \mathrm{g}$ e $\mathrm{n}$ vor, da für tiefergreifende solifluidale Prozesse die Voraussetzungen wie Eislamellen und Eislinsen im Boden bei den kurzen Nachtfrösten nicht gegeben sind. Meist fallen die Temperaturen in der alpinen Stufe des Chirripó nur wenige Grade unter den Nullpunkt; das absolute bisher gemessene Minimum betrug $-9^{\circ} \mathrm{C}$ (KOHKEMPER 1971, am 21. Februar 1971). R a s e n s c h ä l e n an den Rändern von Pfützen und verlandeten Karseen kann stellenweise beobachtet werden. A u f f r i e r u n g e $n$ d e s L o cke r bode n s (Taf. 1, Fig. 4) sind jedoch an oft durchfeuchteten und vegetationsfreien Stellen mit Feinmaterial festgestellt worden. Wo die Pflanzendecke dicht schließt oder Fels ansteht, ist das nächtliche Wachstum von Kammeis ausgeschlossen. 
Die meisten der großflächig entwickelten Formen sind in ihrer Gestaltung somit nicht an das heutige Klima gebunden, sondern älteren Ursprungs.

Die Veränderungs ges chwindigke it des Reliefs in der alpinen Stufe des Chirripó ist sehr klein. Dies steht in großem Kontrast zu den sonst im Land fast überall tiefgreifend und schnell wirkenden rezenten Prozeßgefügen. Relativ flache Hangneigung, dünne Bedeckung mit Feinmaterial, nur schwache Fröste, limitierte Menge abfließenden Wassers in dieser Gebirgslandschaft und nur zögernd einsetzende anthropogene Beeinflussung sind die Erklärung für diese Befunde.

Viel weniger als in den gemäßigten Breiten oder auch in höher aufragenden tropischen Gebirgen wurde die glaziale Bühne verändert. Die rezenten Prozesse haben das kaltzeitlich entstandene Relief nur dekoriert, nicht aber wesentlich umgeformt.

Nach Abschmelzen der Kargletscher und der perennierenden Schneeflecke waren Solifluidalbewegungen an der Hangformung in größerem Umfang beteiligt. Deutlich wird dies zwischen dem oberen Talboden des Valle de Conejos und der Wasserscheide zwischen ihm und dem Valle Ditkebi. Hier sind unter Gras und Buschwerk versteckte $\mathrm{T}$ e $\mathrm{r} r \mathrm{~s} s$ e $\mathrm{t} \mathrm{te} \mathrm{n}$ (sorted steps) vorhanden, die eine Größenordnung von $80 \mathrm{~cm}$ für die Terrassenflächen und $50 \mathrm{~cm}$ für die Terrassenhänge aufweisen und an ihrer Oberfläche eine Anreicherung scherbigen Frostschuttes erkennen lassen. Die Spuren dieser Reliefgeneration werden zwar an dieser Stelle rezent kaum verwischt, sind aber im Arbeitsgebiet sonst nur an wenigen Hängen sichtbar.

Mit präglazialer Anlage, glazialer durchgreifender Formung und rezenter Dekoration kann die Entwicklung des Hochgebirgsteliefs des Chirripó charakterisiert werden.

\section{Schriftenverzeichnis}

Bergoeing, J. P. (1977): Modelado glaciar en la Cordillera de Talamanca, Costa Rica - Instituto Geográfico Nacional, Informe Semestral, 2: 33-44; San José.

BozA, M. \& MENDOZA, R. (1981): The National Parks of Costa Rica. - 310 S.; Madrid (Incafo S. A.).
Chaverri, A. Vaughan, C. \& Poveda, L. (1976): Informe de la gira efectuada al macizo del Chirripó a raiz del fuego ocurrido en marzo de 1976. - 27 S.; Heredia (Universidad Nacional).

HASTENRATH, S. (1973): On the pleistocene glaciation of the Cordillera de Talamanca, Costa Rica - Z. Gletscherkunde und Glazialgeologie, 9, 1-2: 105-121; Innsbruck.

HEINE, K. (1977): Beobachtungen und Überlegungen zur eiszeitlichen Depression von Schneegrenze und Strukturbodengrenze in den Tropen und Subtropen. - Erdkunde, 31, 3: 161-178; Bonn.

- (1983): Ein außergewöhnlicher Gletschervorstoß in Mexico vor 12000 Jahren - ein Beitrag zum Problem der spätglazialen Klimaschwankungen. - Catena, 10: 1-25; Braunschweig.

KOHKEMPER, M. (1971): Expedición de montañeros al Cerro Chirripó encuentra los restos de la avioneta hondureña HR - 268. - Instituto Geográfico Nacional, Informe Semestral, 1: 63-69; San José.

SCHUBERT, C. (1975): Glaciation and periglacial morphology in the northwestern Venezuelan Andes. Dedicated to the memory of Carl Troll. - Eiszeitalter u. Gegenwart, 26: 196-211; Öhringen/Württ.

- (1976): Evidence of former glaciation in the Sierra de Perija, western Venezuela. - Erdkunde, 30: 222-224; Bonn.

Vaughan, C., Chaverri, A. \& Poveda, L. (1976): El macizo de Chirripó está resucitando - La Nación, 14 de diciembre: 5 C; San José.

WeBer, H. (1959): Los páramos de Costa Rica y su concatenación fitográfica con los Andes Suramericanos. 67 S., Instituto Geográfico Nacional, San José.

WeYL, R. (1955): Vistigios de una glaciación del Pleistoceno en la Cordillera de Talamanca, Costa Rica - Instituto Geográfico Nacional, Informe Trimestral, 2: 9-32; San José.

- (1956): Eiszeitliche Gletscherspuren in Costa Rica (Mittelamerika) - Z. Gletscherkunde und Glazialgeologie, 3: 317-325; Innsbruck.

- (1957): Vestigios de los glaciares del Pleistoceno en la Cordillera de Talamanca - Instituto Geográfico Nacional, Informe Trimestral, 1: 36-53; San José.

Manuskript eingegangen am 13.12. 1984 


\section{Tafel 1}

Taf. 1, Fig. 1: Lago San Juan, der größte der Karseen in der alpinen Stufe, gerade westlich des Chirripó Grande.

Taf. 1, Fig. 2: Valle de los Conejos in etwa $3500 \mathrm{~m}$ Höhe. In den Boden des Glazialtrogs ist ein schmaler Bach eingekerbt, der nach Regen episodisch Wasser führt. Dies ist die einzige deutliche Umformung seit der pleistozänen Vergletscherung.

Taf. 1, Fig. 3: Gletscherschliff auf $3550 \mathrm{~m}$ Höhe im Valle de los Conejos.

Taf. 1, Fig. 4: Durch Kammeis aufgefrorener Boden am Fuß des Gipfels Noreste auf $3550 \mathrm{~m}$ Höhe. 

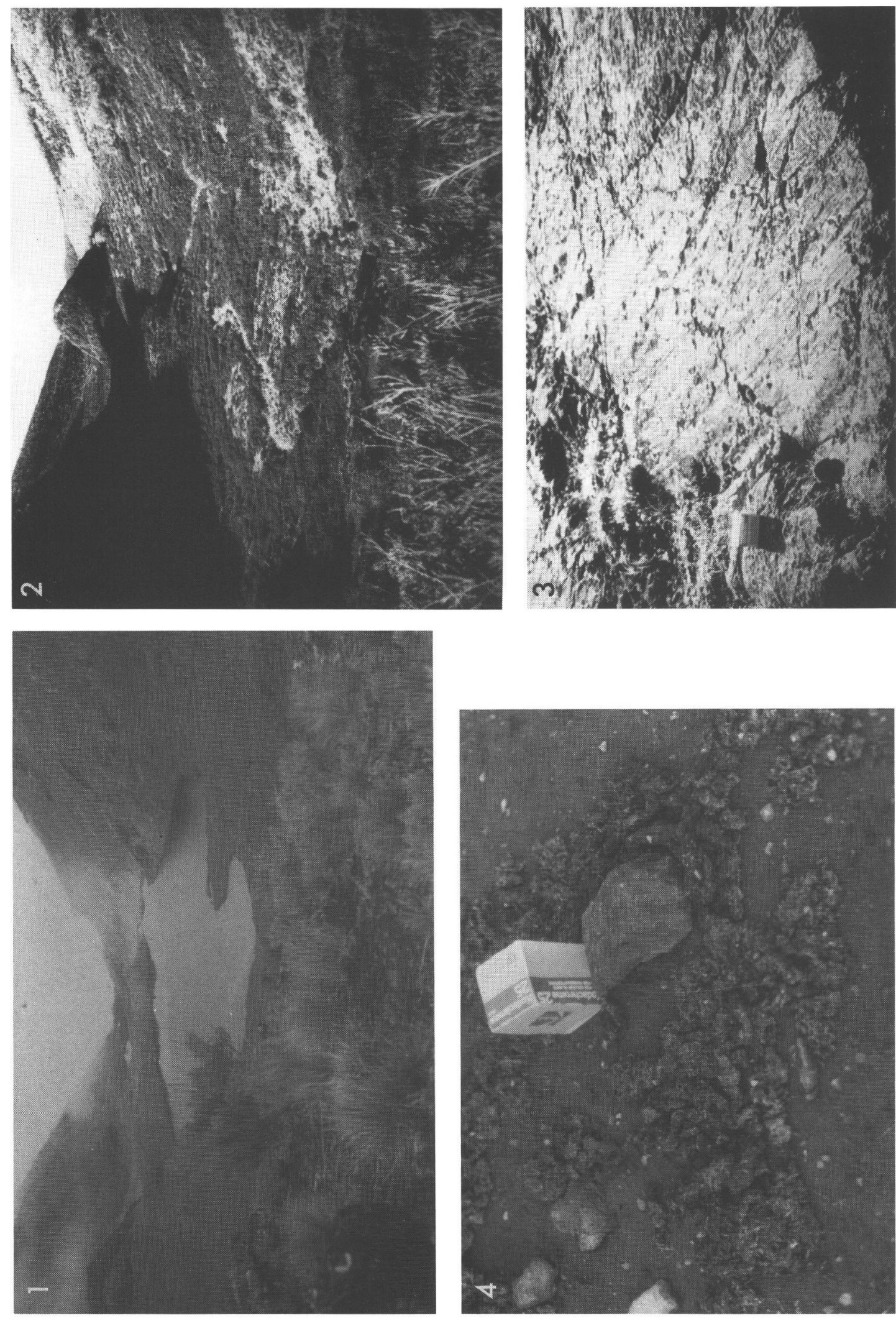
